\title{
LA FORMACIÓN PERMANENTE DEL SACERDOTE EN EL POSTCONCILIO
}

DOI: https://doi.org/10.52039/seminarios.v58i205-206.292

SATURNINO GAMARRA*

Si siempre que se me ha pedido un servicio para los sacerdotes he terminado aceptándolo, creo que en esta ocasión he ido más lejos, hasta he facilitado la misma propuesta: la acogida de mi parte iba surgiendo al mismo tiempo que escuchaba el tema; invitación y acogida han ido a la par. No necesité tiempo para pensarlo. Se me hablaba de algo que tocaba el centro de mi vida y el campo de trabajo de muchos años.

Como el tema admite estudios diversos y enfoques muy diferentes, conviene que señalemos los límites de esta aportación, para que así conozcamos ya, de antemano, el terreno en el que pensamos movernos. No se trata de hacer una historia que presente, de la forma más exhaustiva y con el mayor número de datos posible, lo que ha sido la Formación Permanente de los sacerdotes entre nosotros hasta el día de hoy; sino que pretendemos algo más: partiendo de unos datos básicos y reales, nos interesa acercarnos al presente de la Formación Permanente para que, revisando su pasado, pensemos en su futuro.

Lo que buscamos no es la constatación de unos datos, sino el juicio de valor sobre lo que ha sido la Formación Permanente de los sacerdotes y lo que puede y debe ser en el futuro. En este caso, conviene dar respuesta a una serie de preguntas como: ¿Lo que ha dado la Formación Permanente en el postconcilio es todo lo que se podía haber esperado de ella? ¿Se ajustó bien a todo lo que es la Formación Permanente? ¿Se comprendió bien desde el comienzo su naturaleza? ¿Qué factores fueron los que marcaron la línea que ha seguido hasta nuestros días? Y si pensamos en el futuro de la Formación Permanente, están las preguntas: ¿Sería suficiente con plantear una adaptación de la Formación Permanente, tal como se la ha concebido hasta ahora, a la nueva situación actual? ¿No necesitará un replanteamiento en totalidad?

Resulta obvio que el ideal para este tipo de trabajos es el de presentarse como el resultado de un intercambio de puntos de vista y de un contraste de opi-

* D. Saturnino Gamarra, un enamorado de la formación sacerdotal. Actualmente Director de la Revista Surge. Pertenece al Consejo de consultores de Vitoria. Profesor emérito de la Facultad de Teología del Norte de España (sede Vitoria) y Director espiritual de su Seminario. 
niones sobre la Formación Permanente; pero nuestra aportación va a carecer de esta confrontación, y lamentamos la ausencia. Dejaremos el valor de esta aportación en su carácter testimonial, es decir, en ser una llamada de atención en solitario sobre la Formación de los sacerdotes.

\section{Acercamiento a lo que ha sido la Formación Permanente}

El acercamiento que pretendemos puede sernos a todos fácil y cómodo; el camino que vamos a recorrer nos resultará bastante conocido, pues se trata de algo que es actual y que no es tan antiguo; sólo cuenta con el hoy y con un ayer.

\section{El «iter» de la Formación Permanente del sacerdote}

El «iter» que ha seguido la Formación Permanente tiene la misma orientación desde el comienzo; nos será fácil llegar a una síntesis breve de su trayectoria ${ }^{1}$.

a) Vaticano II. La llamada a proseguir y a completar la formación de los sacerdotes después del Seminario está muy presente en el Vaticano II. Son tres los textos que se refieren explícitamente a nuestro tema: OT 22; PO 19; CD 16. En su tratamiento existe una gradación: si en OT se plantea la necesidad de proseguir y completar la formación sacerdotal en razón de «las condiciones de la sociedad moderna», en PO se acentúa la urgencia de una mayor preparación, y por esta razón insta a utilizar medios para ello, y en CD se señala la responsabilidad que tienen los Obispos sobre la vida y la misión de los sacerdotes.

Y el planteamiento también es diverso según el contexto en el que se trata la Formación Permanente. Así, OT dedica el apartado VII con un único número, el 22, para presentar el «Perfeccionamiento de la Formación después de los estudios $»^{2}$; PO dedica dos párrafos al tema dentro del número 19 , orientado al estudio y a la ciencia pastoral de los presbíteros; y CD 16 sitúa el tema dentro del deber que los Obispos tienen de regir y apacentar a sus fieles. Los matices, pues, son distintos. La Formación Permanente en OT tiene un carácter de formación; en PO su nota característica es más bien de capacitación para la pastoral; y en CD es vista desde la responsabilidad del Obispo.

1. La bibliografía sobre la Formación Permanente del sacerdote es muy abundante. La revista Surge, además de artículos sueltos, tiene dos números monográficos sobre la Formación Permanente de los sacerdotes: Surge 31 (1973) 437-520; 43 (1985) 293-363; la revista Sal Terrae también dedica al tema dos números monográficos: Sal Terrae 67 (1979) 3-39; 69 (1981) 755-825; y Seminarios, que no dedica un número monográfico al tema, en muchos de sus artículos estudia la formación de los sacerdotes. Entre los muchos artículos que se han escrito, citamos: L. A. Montes-J. A. Ubieta, Los presbiteros y la Formación Permanente a partir del Vaticano II en nuestra Iglesia, en Comisión Episcopal del Clero, La Formación Permanente de los sacerdotes. Simposio, Madrid 1993, 31-79.

2. El contenido de este número de OT no es algo aislado ni un añadido a última hora, sino que está muy dentro del Documento, ya que está presente en el primer esquema de 1963. Cf. ASyn III, VII, 803. 
b) Inter ea, «Carta circular de la Sagrada Congregación del Clero a las Conferencias Episcopales sobre la Formación Permanente de los sacerdotes, especialmente de los jóvenes». Es el primer documento oficial dedicado al tema. Su fecha es el 4 de Noviembre de $1969^{3}$. Llega en un momento especialmente crítico y convulso del sacerdocio en la Iglesia -recordamos el Congreso sacerdotal de Lucerna (Suiza) en septiembre de 1967, el Congreso de formación sacerdotal de Metz (Francia) en abril de 1969 y la Asamblea europea de sacerdotes de Chur (Suiza) en Julio de 1969, que apoyaron una secularización radical del sacerdocio con enfrentamientos en la Iglesia ${ }^{4}-$, y la Carta pretende, además, dar respuesta a la problemática que recoge de la consulta que la misma Congregación acababa de hacer a las Conferencias Episcopales. La carta circular deja asentado el principio de formación estableciendo la relación que debe darse entre los tres aspectos -espiritual, intelectual, pastoral-: «Esta vida espiritual hay que considerarla como el fundamento de los otros dos aspectos (intelectual y pastoral), ya que la actividad pastoral es como su fruto y la ciencia teológica su criterio orientador», y, a continuación, pone el énfasis en los medios que puedan garantizar la formación en este momento de confusión.

c) La Ratio fundamentalis Institutionis sacerdotalis (1970). Este Documento de la Congregación de la Enseñanza Católica que contiene las «Normas Fundamentales de la Formación del sacerdote» dedica el capítulo XVII, también el último, como OT, al tema: «La Formación hay que completarla después del Seminario». Es el principio en el que se insiste una y otra vez, y con el cual hay que contar.

d) La recepción de la Formación Permanente en el año 1970. Resulta llamativo que el Secretariado nacional del Clero pueda presentar para el verano de 1970 este programa de cursos: «IV Coloquio de Teología Moral», en El Escorial; «Convivencia bíblico pastoral», en Bechi (Castellón); «Cursos de Aggiornamento para sacerdotes», en Deusto (Bilbao); "Curso de ciencias sociales», en Madrid; «Curso para formadores de sacerdotes», en Salamanca; "Curso de pastoral social», en Málaga; "Curso especial de renovación conciliar para animadores de comunidades cristianas», en Segovia; «Curso de renovación teológica» en Comillas (Santander); «Curso de renovación teológica», en Zaragoza; «Dirección espiritual y sexología», en Málaga; «Teología y espiritualidad del sacerdocio», en Burgos; «Problemática teológica en torno a la secularización», en San Cugat del Vallés (Barcelona); «Reflexión pastoral según el Evangelio», en Madrid; «El sacerdocio ministerial en el marco de la misión de la Iglesia», en Pamplona; «Ejercicios espirituales de mes para sacerdotes», en Pedreña (Santander) $)^{5}$. Estos datos son de por sí claros exponentes de una abundante oferta de Formación Permanente que se hace a los sacerdotes.

3. AAS 62 (1970) 123-134; Ecclesia 30 (1970) 237-241.

4. S. Gamarra, "La secularidad en las Escuelas de Espiritualidad Sacerdotal», en Comisión Episcopal del Clero, Presbiterado y Secularidad. Simposio, Madrid 1999, 304-305.

5. Cf. Surge 30 (1970) 147-156. 
e) La Formación Permanente en la «Asamblea Conjunta. Obispos-Sacerdotes» (septiembre 1971). En medio de una búsqueda inquieta y ansiosa se celebra la Asamblea Conjunta en el mes de Septiembre de $1971^{6}$. Hay que situarla entre los congresos de Lucerna, Metz, Chur, ya señalados, y el encuentro sacerdotal de Ginebra (abril 1971) y el Sínodo de los Obispos que se celebraría dos meses después en Roma, durante el mes de octubre hasta el 6 de noviembre de 1971, con dos temas: «La justicia en el mundo» y «El sacerdocio ministerial», adoptando sobre la identidad del sacerdocio, tema clave en esta época, una postura firme y clarificadora. Se puede dudar de si la fecha de la Asamblea Conjunta fue la apropiada para poder llegar a una reflexión serena y compartida sobre temas de tanta envergadura ${ }^{7}$.

La Asamblea contó con la ponencia VII sobre «La preparación para el sacerdocio ministerial y formación permanente del clero», que fue muy bien acogida por los asistentes. Con todo, se puede apreciar, en primer lugar, que en ella predomina el estudio del cambio socio-cultural para plantear que tanto la respuesta del ministerio como la formación permanente deben hacerse prioritariamente desde los postulados de dicho cambio; $y$, en segundo lugar, que «plantea la formación permanente desde tres niveles: nivel personal, nivel doctrinal y nivel pastoral ${ }^{8}$, lo cual hace ver que se obvia incluir el aspecto espiritual, que hasta ahora se había presentado como el fundamental. ¿Por qué?

f) Las ofertas de Formación Permanente se consolidan. Recogemos del artículo, ya citado, de L. A. Montes Peral - J. A. Ubieta, algunas de las experiencias de Formación Permanente que se han dado. El «Centro Regional de Formación Permanente del Clero» de Aragón, creado en 1970. El «Curso de Actualización Teológico-Pastoral», de un año de duración, fue ofrecido por la Facultad de Teología de Vitoria en colaboración con las Vicarías de Pastoral de las diócesis vascas y Navarra, y por el que han pasado desde el curso 1978-1979 al curso 19961997, que fue el último, 508 alumnos $^{9}$. Los «Cursos de renovación sacerdotal» en Andalucía, de un mes de duración durante el verano, desde 1978 hasta 1989. El «Curso Diocesano» de Badajoz, de varias semanas, entre los años 19852003. El curso de «Renovación sacerdotal en la Región del Duero», de un mes de duración, que tuvo ocho celebraciones, siete en Salamanca (1991-1997) y una en Burgos $(2001)^{10}$. Tiene un relieve especial el «Curso de renovación sacerdotal», creado por la Comisión Episcopal del Clero, en colaboración con la

6. Secretariado Nacional del Clero, Asamblea conjunta. Obispos-sacerdotes. Madrid 1971.

7. Merece la pena entrar en el estudio de esta Asamblea, un tanto compleja. Su bibliografía es tan abundante como diversa y hasta opuesta. Llamamos la atención del alcance que tuvo.

8. Id., 577-578.

9. Cf. A. M. Navarro, Actualización teológico-espiritual: balance y posibilidades de una experiencia: Surge 43 (1985) 295-311.

10. El Equipo Regional de Formación Permanente del Duero sigue reuniéndose dos o tres veces al año desde el 1980. 
Universidad Pontificia de Salamanca, que ha venido celebrándose desde el curso 1985-86 al curso 2010-11. Deben tenerse muy en cuenta la «Formación Permanente in situ», que ha tenido y tiene aplicación en diversos lugares ${ }^{11}$, y el servicio que ha ofrecido durante una larga época el «Instituto Internacional de Teología a Distancia» ${ }^{12}$. Merecen también una mención especial las revistas y publicaciones teológicas de las Facultades, de los Centros Teológicos y de los Seminarios por su aportación a la Formación Permanente. Y no puede olvidarse la praxis del Año sabático que, al constatarse su eficacia, está haciéndose muy habitual.

g) La Formación Permanente en el Código de Derecho Canónico (1983). El canon 273 no sólo recoge el espíritu que se respiraba en torno a la formación de los sacerdotes, sino que precisa su contenido y urge su cumplimiento. La Formación Permanente entra ya dentro de las obligaciones y los derechos de los clérigos.

h) La Formación Permanente del Simposio y del Congreso de Espiritualidad Sacerdotal. El «Simposio de Espiritualidad del presbítero diocesano secular», celebrado en Madrid, del 30 de octubre al 2 de noviembre de $1986^{13}$, y el «Congreso de Espiritualidad Sacerdotal», celebrado también en Madrid, del 11 al 15 de septiembre de $1989^{14}$, fueron un hito clave en la Formación Permanente de los sacerdotes en la Iglesia postconciliar en España. Es verdad que ni el Simposio ni el Congreso hablaron de forma expresa de la Formación Permanente, pero abordaron un punto fundamental para dicha formación, que hasta ese momento fue aparcado, que es la identidad del sacerdote y su espiritualidad. La singularidad de esta aportación no estuvo sólo en plantear la identidad del sacerdote sino, además, en presentar la espiritualidad en su misma identidad. Es desde esta base cuando se pudo pensar ya en una Formación Permanente Integral del sacerdote. Sin la referencia a la identidad y a la espiritualidad del sacerdote no tendremos una formación integral, faltaría su identificación.

i) «Simposio de la Formación Permanente de los sacerdotes» (1992). La Comisión Episcopal del Clero organizó un «Simposio sobre la Formación Permanente de los sacerdotes» que se celebró en los días 4 al 7 de diciembre de 1992. ¿Se acertó en la fecha? Démonos cuenta de que en el año 1990 tuvo lugar el Sínodo de Obispos en Roma sobre «La Formación de los sacerdotes en las circunstancias actuales» y de que acababa de publicarse la Exhortación postsinodal Pastores dabo vobis (1992), que dedica el capítulo VI a la Formación Permanente

11. Siguen esta forma diversas diócesis como Badajoz, Burgos, Palencia, Orense, Valencia, entre otras, aunque con algunas señales de decaimiento. Cf. J. Tapia, La Formación Permanente en el lugar del trabajo pastoral del presbitero, en Comisión Episcopal del Clero, La formación permanente..., 273-289.

12. Cf. A. García-Gascó, Un modo de afrontar el problema de la Formación Permanente, El Instituto Español de Teología a Distancia: Surge 31(1973) 456-467.

13. Comisión Episcopal del Clero, Espiritualidad del presbítero diocesano secular. Simposio. Madrid 1987.

14. Comisión Episcopal del Clero, Espiritualidad sacerdotal. Congreso, Madrid 1989. 
de los sacerdotes. ¿Nuestro Simposio tuvo tiempo para asimilar lo que se le ofrecía desde el Sínodo de Roma y desde la Exhortación Pastores dabo vobis sobre la Formación Permanente, objeto de su reflexión? ¿Encontró espacio para ofrecer el resultado de sus reflexiones? ¿Han tenido eco sus propuestas?

No cabe ninguna duda de que «Las propuestas del Simposio a la Comisión Episcopal del Clero», que fueron 14, tuvieron un carácter propio. Ante la variedad amplísima de experiencias de Formación que se había dado, se experimentó que urgía tener una buena orientación organizativa; se precisó lo que es la Formación integral permanente al mismo tiempo que se reconocía su necesidad; también se aceptó que había que llegar a la institucionalización de esta Formación, aunque no se llegó a ninguna concreción ${ }^{15}$.

j) La Formación Permanente en Pastores dabo vobis (1992). Es algo comúnmente aceptado que hoy, cuando se habla de la Formación Permanente de los sacerdotes, la referencia obligada es la Exhortación Apostólica postsinodal de Juan Pablo II, Pastores dabo vobis. Ésta dedica el capítulo VI al tema con 12 números, del 70 al 81 , de hondo contenido ${ }^{16}$. Las perspectivas que nos ofrece son muchas y novedosas; en este momento nos limitamos a resaltar los siguientes aspectos: 1) Salta a la vista el subrayado que se da a las razones teológicas para la Formación Permanente del sacerdote, porque estábamos acostumbrados a que se nos presente ésta desde una perspectiva meramente humana; y llama la atención que el punto de partida de esta formación sea hoy la identidad del presbitero. Cruza el documento la opción de ver «La identidad presbiteral como principio estructurador e integrador de la persona del sacerdote» (cf. PDV 11). 2) La caridad pastoral -dentro de la identidad-es «alma y forma de la Formación Permanente del sacerdote» (PDV 70). 3) Se tiene muy en cuenta el proceso de la persona y de la vida del sacerdote; y se habla de un continuum entre el Seminario y la vida del sacerdote (PDV 71). 4) Se plantea la integralidad de la formación incluyendo cuatro dimensiones: la humana, la espiritual, la doctrinal y la pastoral.

Conviene recordar que la referencia de la Formación Permanente a Pastores dabo vobis tiene hoy plena vigencia; que no es un documento obsoleto, sino que está pidiendo que se siga contando con él ${ }^{17}$. La referencia a él es ineludible.

k) La formación Permanente a partir de Pastores dabo vobis. La Comisión Episcopal del Clero, a partir de Pastores dabo vobis y sin dejar su mano, ha he-

15. Cf. S. Gamarra, Presentación y comentario de las Propuestas del Simposio sobre la 'Formación Permanente de los sacerdotes: Surge 51 (1993) 201-217.

16. Cf. S. Gamarra, La Formación Permanente de los sacerdotes en «Pastores dabo vobis»: Surge 51 (1993) 418-445; R. Sánchez Chamoso, La Formación Permanente: Seminarios 38 (1992) 401-419.

17. En 2005 la revista Surge dedicó un número a los 13 años de Pastores dabo vobis, contando con 26 aportaciones de especialistas, según un guión previo, sobre la valía y la actualidad del Documento. El balance ha sido unánimemente positivo. Cf. Surge 63 (2005) 1-200. 
cho un gran servicio a la Formación Permanente de los sacerdotes con publicaciones y asambleas de Delegados del Clero. El punto de partida estuvo en el Documento de los Obispos de la Comisión, titulado Sacerdotes, día a día. La formación permanente integral (1995). Le siguieron otras publicaciones: La formación humana de los sacerdotes según «Pastores dabo vobis» (1994), de Juan María Uriarte y Juan Luis Ruiz de la Peña; La formación espiritual de los sacerdotes según «Pastores dabo vobis» (1995), de Lorenzo Trujillo, Juan María Uriarte y Alfonso Crespo; La formación intelectual de los sacerdotes según «Pastores dabo vobis» (1996), de Olegario González de Cardedal y José Manuel Sánchez Caro; La formación pastoral de los sacerdotes, según "Pastores dabo vobis» (1998), de Julio Ramos Guerreira y Bernardo Álvarez Afonso ${ }^{18}$. Estas aportaciones que, sin duda alguna, fueron unas llamadas muy esperanzadoras para su momento, no dejan de ser hoy llamadas inquietantes ante el futuro.

I) Experiencias de Formación Permanente más recientes. Nos referimos, en primer lugar, al «Curso de Actualización Sacerdotal» que ofrece el Colegio Español de Roma a los sacerdotes de todas las diócesis de España, como Formación Permanente. Es un curso de un mes de duración en Roma, en tiempo de Pascua, entre los meses de abril y mayo; con el de este año, 2012, se llega a la XXV edición. También nos referimos al curso que ofrecen los Jesuitas en Manresa (Barcelona): «Dos meses de reciclaje». Todos los profesores son miembros del Centro de Estudios "Cristianisme i justicia», y está destinado a laicos y laicas, jesuitas, religiosos y religiosas, y sacerdotes (entre 35-75 años). El curso de este año comienza el 22 de enero y termina el 27 de marzo. Dura, por tanto, nueve semanas. Tenemos, a última hora, el anuncio del curso «Máster, actualización teológica y formación permanente», siguiendo los nuevos cursos y actividades del Instituto Superior de Pastoral, que se imparten en el Campus de la UPSA en Madrid.

\section{Un juicio de valor}

Una vez que hemos recorrido con mirada comprensiva y atenta el camino que ha seguido la Formación Permanente de los sacerdotes en el postconcilio, y sintiéndonos, por otro lado, muy dentro de la vida y de la formación de los curas, nos atrevemos a dar un juicio de valor sobre esta Formación Permanente. Este atrevimiento no es tan atrevido como pudiera parecer, tiene su base: si tuvimos parte en el proceso de la Formación Permanente, y somos testigos de la situación que estamos viviendo hoy los presbíteros diocesanos, podremos preguntarnos con todo derecho: ¿La Formación Permanente que llega hasta nosotros vale para nuestro hoy?

18. Estos documentos han sido recopilados y publicados en un volumen: Comisión Episcopal del Clero, La formación sacerdotal permanente, Madrid 2004. 
a) Un recorrido con buena nota

Lo realizado a lo largo de estos años merece una muy buena calificación. La apuesta a favor de la Formación Permanente ha sido firme y constante durante muchos años; los esfuerzos realizados han sido grandes; el material que se ha ofrecido es valioso y de gran calidad; se ha impuesto una mentalidad a favor de la Formación Permanente; se ha podido llegar a conocer con más precisión lo que es dicha Formación; la impresión de los que han participado en los cursos es normalmente satisfactoria o muy satisfactoria; y son muchos los servicios de Formación que se han ofrecido a los sacerdotes en estos años, con una fuerte inversión económica incluida.

Pero aunque los resultados sean claramente positivos, ¿son del todo satisfactorios? Nos parecen insuficientes.

\section{b) Puntos de insuficiencia}

La sospecha sobre el valor real de la Formación Permanente se nos cuela dentro de nosotros mismos cuando contemplamos la situación sacerdotal en la que nos movemos. También es verdad que debemos ser críticos con la misma sospecha, y por eso nos preguntamos: ¿Es correcto que ahora pongamos todos nuestros males en el debe de la Formación Permanente? Que se reaccione de esta manera tiene su explicación: porque si se puso la solución de todo lo sacerdotal en la Formación Permanente, es normal que ahora nuestros fallos se los carguemos a ella. Sin caer en extremismos, nos preguntamos de corazón: ¿Hemos llevado bien la Formación Permanente? ¿Qué hemos perdido durante el camino? ¿Hemos dejado de lado algo que, siendo fundamental, debíamos haber tenido en cuenta? Nos referimos a las insuficiencias que ha podido tener la Formación Permanente entre nosotros:

-Mayor realismo. Durante muchos años, desde el Vaticano II, cuyo final fue en Diciembre de 1965, se ha seguido hablando con acentos distintos, pero siempre fuertes, de la necesidad de la Formación Permanente de los sacerdotes y de su urgencia, tal como lo hemos visto en las páginas anteriores, pero han faltado evaluaciones diocesanas, regionales y de carácter nacional y no se ha contado con datos, al menos ofrecidos al común de los sacerdotes. Se sabe que la Formación Permanente no llega a todos, ni mucho menos, y que cuando ésta se entiende como la participación en algún curso, ya no se repite, argumentando: «iYo ya hice la 'Formación Permanente'!». ¿Cabe una expresión más dislocada?

Registramos que el Documento Sacerdotes, día a día, de la Comisión Episcopal del Clero, hace este comentario de la realidad, que, aunque sea del año 1995, puede servirnos de pauta: «Digámoslo claramente: a pesar de todos los esfuerzos de las diócesis, de sus delegaciones para el clero y de otras instituciones, la respuesta a las ofertas diocesanas oscila entre un $30 \%$ y un $40 \%$. Debemos preguntarnos seriamente qué nos está pasando a un $60 \%$ o $70 \%$ res- 
pecto a la formación permanente. Un número tan elevado de ausencias revela una desmotivación de alto riesgo. Es verdad que suelen darse a veces respuestas personales de formación permanente, al margen de los ofrecimientos diocesanos. La impresión, sin embargo, es que estas respuestas personales son escasas y reducidas ${ }^{19}$.

Pensamos que la Formación Permanente que se ha ofrecido en esta época ha querido ir, a base de voluntad, más allá de la realidad, sin ajustarse a ella, y que los resultados no le han acompañado como eran de esperar. La visión de la realidad debería haber sido tomada más en cuenta.

-Atención a los objetivos. Consideramos que un punto decisivo para evaluar la Formación Permanente es ver los objetivos que han inspirado cada una de las actividades realizadas para la formación del sacerdote. Porque no es lo mismo el objetivo de actualizar la formación recibida antes del Vaticano II y el objetivo de capacitarse para responder a las nuevas situaciones que vayan surgiendo; como tampoco son lo mismo el objetivo de un curso de adoctrinamiento, el de un curso de capacitación pastoral y el de un curso de formación integral.

Creemos que una clarificación ajustada del fin de la Formación Permanente es del todo necesaria. Sabemos que la Formación Permanente es integral, pero, de hecho, se ha primado el objetivo doctrinal y el pastoral sobre el de la formación integral. La ausencia de la formación integral, en la praxis, ha sido notable.

-La integralidad de la formación. Todos nos damos cuenta de cómo la integralidad ha ido abriéndose camino dentro del proceso que ha seguido la Formación Permanente. Se ha hablado de ella con toda normalidad. Pero, ¿cómo se obtiene? ¿Se obtiene por hacer un reparto equitativo de materias: dogmática, bíblica, pastoral, espiritual? ¿La integralidad es una yuxtaposición de materias? ¿La integralidad está sólo o principalmente en lo académico? ¿No está en dar con el factor integrador de toda la persona en formación, que en nuestro caso es la misma identidad del sacerdocio?

Parece que lo habíamos tenido fácil, porque la crisis de identidad sacerdotal había remitido y se nos habían ofrecido elementos firmes de nuestra identidad en el Sínodo de Obispos de 1971, en el Simposio y en el Congreso de Espiritualidad sacerdotal, y especialmente en PDV; pero, a pesar de todo, se ha seguido el camino que viene desde el comienzo, que no es otro que el de de una formación doctrinal y pastoral. No se ha registrado un cambio reseñable de postura a favor de una mayor integralidad.

-La conciencia de la obligatoriedad. Es bastante común pensar que la Formación Permanente, identificada con cursos de profundización y de actualización teológico pastoral, es de libre elección; está en uno decidirse a cursarlos o no. Y

19. Comisión Episcopal del Clero, La Formación Sacerdotal Permanente, Madrid 2004, 131. 
en el caso de que se decida a su favor, uno es el que elige la Formación entre las distintas ofertas que se presentan. Y como está en uno la elección, es uno mismo quien elige el momento, el tiempo de duración, el lugar, el profesorado y el cómo hacerla. En una palabra, se puede decir que es una formación a la carta. ¿Debe ser así? De hecho, esta forma de funcionamiento se ha dado y se da.

Este es el hecho que se repite, aunque todos sepamos que la obligatoriedad está más allá de las propias apetencias y de que esté mandado. Sabemos desde lo que nos han enseñado estos años: que la verdadera Formación descansa en la misma identidad sacerdotal, en su honda vivencia; que le es imprescindible la relación estrecha, es decir que el continuum sea real entre el Seminario y el postseminario (PDV 31); y que necesita el apoyo de una institucionalización concreta y muy asumida.

Sobre el punto de si la conciencia de una obligatoriedad por la Formación Permanente ha ido a más entre los sacerdotes a lo largo de estos años, nos parece que no va mucho más allá de saber que se cuenta con la posibilidad de hacer unos cursos en la medida en que uno los necesite y cuando los necesite.

-El acompañamiento. Todos sabemos que la formación incluye un acompañamiento, y también lo incluye la Formación Permanente de los sacerdotes. La formación autodidacta en el sacerdocio no puede ser aconsejable, ya que ser discípulo, que afecta a la manera de ser, es connatural a ser sacerdote de Jesucristo. Para comprender este acompañamiento de la Formación Permanente integral, debemos verlo en el sacerdote, destinatario de la formación, en el que acompaña o formador y en las formas del acompañamiento.

Si miramos al sacerdote, destinatario o sujeto de la formación, vemos que en ella se ha contado mucho con la persona hasta darle una plena libertad de movimientos, pero en cambio no se ha atendido a la persona. Lo que se ha primado en nuestra Formación Permanente ha sido la formación en grupo, y ha faltado la formación personalizada.

Si miramos al acompañante o formador en la Formación Permanente de los sacerdotes que se ha llevado a cabo, lo que descubrimos es más su ausencia que su presencia. ¿Quién acompaña para marcar los objetivos a tener en cuenta en el curso que va a realizar, para integrar lo que va recibiendo en él y para programar los pasos siguientes?

Si miramos las formas del acompañamiento en el tipo de formación que se ha seguido, vemos que no se ha pensado en una Formación Permanente sin grupo ni cursos. ¿La formación debe contar necesariamente sólo con cursos? ¿No se puede pensar, además, en una Formación Permanente integral más personalizada, al estilo de una tutoría, sin tener que recurrir a cursos?

El acompañamiento que ha funcionado en nuestra formación ha resultado escaso y poco directo. Presentamos un tipo de acompañamiento que entre nosotros resulta común: el sacerdote de más confianza, a quien le comunica la 
decisión, le ayuda a la elección del curso y espera pacientemente a que lo termine para valorar los resultados. Si nuestro acompañamiento ha sido así, no deja de ser insuficiente.

Llegamos al final de la primera parte. Aunque nos hemos extendido en ella más de lo que habíamos pensado, mantenemos nuestro objetivo inicial: Una vez conocida la trayectoria que la Formación Permanente ha seguido entre nosotros, pensamos en su futuro.

\section{Ante la Formación Permanente del Futuro. Un nuevo Planteamiento}

Abordamos esta parte conscientes de la complejidad del tema. Pensamos que no nos anima ningún espíritu de improvisación; y lo que deseamos, teniendo muy presente la Formación Permanente de los sacerdotes -porque la valoramos-, es descubrir sus nuevas posibilidades y ver su aplicación a la realidad en la que nos encontramos. Este es nuestro objetivo.

\section{Dos presupuestos}

De entre los muchos aspectos que deberían tenerse en cuenta, nos limitamos a dos, a los que en este momento consideramos más fundamentales para el futuro de la Formación Permanente.

\section{a) Un más de realismo}

Si se piensa en una Formación Permanente real, es decir que responda al momento presente de los sacerdotes, hay que partir de una visión realista de nuestra situación. Una falta de ajuste entre la formación que se presente y la situación que se viva inutilizaría los esfuerzos que se pongan y haría estéril el nuevo planteamiento. Porque queremos lo mejor para la Formación Permanente, exigimos realismo en su planteamiento.

Y como cae por su peso que la situación real concreta sólo puede verse desde el lugar donde uno se encuentra, lo que ofrecemos sólo tiene un valor indicativo. Nuestra aportación no tiene otro sentido que ofrecer lo que pensamos por si cabe algún trasvase y pueda tener alguna aplicación.

De hecho, la praxis de la Formación Permanente que ha llegado hasta nosotros se encuentra afectada, quizás bloqueada, por varios factores que señalamos a continuación.

-Reducción de número. Esta reducción de número es un hecho claro para todos, y en algunos lugares, clamoroso, aunque intentemos ocultarlo. Basta con que miremos en nuestras diócesis el número de seminaristas que hay, los sacerdotes jóvenes que tenemos y el respetable número de sacerdotes mayores que nos acompañan. Con una reducción de número, que es bastante generalizada, 
se explica que las ofertas de cursos se reduzcan y que los cursos que contaron con una gran aceptación como el Curso de Actualización de Vitoria y el de Salamanca ya no se impartan. Continúan, con las características que le acompañan, el Curso de Actualización de Roma, y el de Manresa, y está la nueva oferta de la Universidad Pontifica de Salamanca en Madrid.

También es verdad que a pesar de dicha reducción se intenta seguir con mínimos -de número y de capacidad-el plan de siempre, el de organizar cursos, jornadas, asambleas, incluyéndolos como Formación Permanente. ¿Qué explicación tiene este empeño? Sin entrar en el análisis del porqué, nos preguntamos: ¿No será, quizás, el momento de repensar la Formación Permanente?

- La diversidad de planteamientos del sacerdocio y de su espiritualidad. Ese «más de realismo» que se nos pide, nos lleva a tomar conciencia de que la diversidad de planteamientos de sacerdocio y de su espiritualidad son ya una realidad, los tenemos entre nosotros. No hablamos de conjeturas, ya que los mismos hechos lo evidencian: la diversidad de planteamientos responde a los tipos de grupos o agrupaciones de sacerdotes que ya existen. Esto, además, nos obliga a ver que la formación que subyace en cada visión del sacerdocio y de su espiritualidad, y que la sostiene, es distinta.

Al hilo de esta situación que estamos describiendo, se nos impone un paso más: cada tipo de formación sacerdotal está necesitando su tipo de Formación Permanente, como vemos que lo practican los grupos que conocemos. Está claro: a cada formación, su Formación Permanente. ¿A qué nos llevará todo esto? No perdamos de vista la realidad a la se puede llegar: si los diversos tipos de formación van en aumento y se llega a una situación en la que no existe una formación sacerdotal común, ni tampoco hay conciencia de que se comparte una misma formación, ya no se necesita una Formación integral Permanente común, sino que la Formación que se va a necesitar o se está necesitando ya es peculiar. Podrán ofrecerse cursos y encuentros de contenidos generales que sirvan para los sacerdotes de los estilos más diversos, pero lo que es la Formación integral Permanente con un carácter personal, porque llega a lo nuclear de la persona, y general, es decir, que tiene un denominador común para todos, ya no tendrá cabida; en cambio, esa formación sólo será posible en cada tipo de sacerdocio y de espiritualidad que se viva en los grupos o agrupaciones. ¿Qué futuro tiene, pues, entre nosotros la Formación integral Permanente?

No defendemos, en absoluto, la situación que describimos; tan sólo constatamos tendencias con alguna de sus consecuencias; pero lo que verdaderamente nos interesa ahora es que la Formación integral Permanente sea eficaz en los sacerdotes, y que para ello no ignore la realidad que existe, sino que cuente con ella. La Formación integral Permanente de los sacerdotes diocesanos es hoy más importante y necesaria que nunca. Llamamos la atención sobre esta urgencia. 
-El debilitamiento o la fragmentación de los presbiterios, como peligro. Sin dejar ese «más de realismo» en el que estamos situados para garantizar un nuevo planteamiento de la Formación Permanente de los sacerdotes, apuntamos tímidamente el riesgo de un debilitamiento y hasta de fragmentación del presbiterio. Lo aducimos solamente para situar bien, en una panorámica amplia, la misma Formación Permanente. Pensamos que la mera situación de amenaza o de peligro de un debilitamiento o de una fragmentación de los presbiterios debe ser punto de partida en la Formación Permanente del sacerdote diocesano, y pensamos también que la superación y la sanación deban ser su objetivo.

\section{b) La aceptación de los medios en la espiritualidad sacerdotal}

Un segundo presupuesto necesario para asegurar hoy un nuevo planteamiento de la Formación Permanente es que los sacerdotes acepten los medios y la Formación Permanente entre ellos. La aceptación de los medios es un tema clave para la vida y la espiritualidad del sacerdote. Su presentación teórica doctrinal es fácil, pero su praxis cuenta con resistencias, que vienen de atrás.

El Vaticano II, en PO 12 y 13, presentó para el presbítero una santidad propia, relacionada con el ministerio. Este planteamiento fue acogido con entusiasmo. Se reconocía que la espiritualidad del sacerdote, partiendo del ministerio, tenía entidad propia y no necesitaba recurrir a otras espiritualidades o formas de entender la vida del sacerdote. La equivocación de muchos de los presbíteros estuvo en aquel momento en pensar que aceptar el ministerio como fuente de espiritualidad suponía la exclusión de los medios; cuando la realidad es otra muy distinta: podrán rechazarse los medios que no partan de la identidad sacerdotal, del ministerio, pero no se puede prescindir de los medios relacionados con la misma identidad sacerdotal. Estos medios son totalmente necesarios. Reconocemos el hecho de que, a pesar de todo, la aceptación de los medios entre los sacerdotes ya va abriéndose camino; esto es verdad, aunque, a nuestro juicio, sin mucha velocidad.

¿Y qué relación tiene la Formación Permanente con los medios? ¿Puede considerarse un medio más? Podemos decir que la Formación Permanente no se puede reducir a medio, es más que medio, ya que tiene sentido en sí misma: «encuentra su propio fundamento y su razón de ser en el dinamismo del sacramento del Orden» (PDV 70); pero también es medio, porque es utilizada en razón de la formación y del perfeccionamiento de la persona. La Formación Permanente no se reduce a medio y es también medio. Se impone hoy su aceptación; no se puede dar un paso en el proceso del sacerdote sin ella. Llegamos a afirmar que la Formación Permanente está inserta en el mismo dinamismo del ser sacerdote (PDV 70) y es un medio urgido por el mismo ministerio.

Uno de los puntos delicados que la Formación Permanente tiene hoy planteado es su institucionalización. El Simposio de la Formación Permanente (1992) 
afirmó en la Propuesta 6: «Así como del Concilio de Trento nacieron los Seminarios para la formación al sacerdocio, del Concilio Vaticano Il podrían nacer las instituciones para la formación en el sacerdocio» ${ }^{20}$; vio la necesidad, pero la propuesta no llegó a más, ni tampoco, después. La obligatoriedad de la Formación Permanente de los sacerdotes, tan clara doctrinalmente y tan existencialmente sentida, debe contar con el apoyo institucional correspondiente.

\section{El discipulado y la Formación integral Permanente}

El tema del discipulado es muy conocido para todos nosotros, pero aquí queremos situarlo en el marco de la Formación integral Permanente de los sacerdotes. Y como pensamos que en nuestro caso la relación entre el discipulado y la formación es más estrecha de lo que a primera vista podría parecernos, levantamos la voz para que el nuevo planteamiento de Formación integral Permanente no pase por alto el discipulado, que es la actitud más básica y necesaria en dicha formación.

\section{a) Un primer acercamiento}

Para evitar un desarrollo prolijo, sabiendo que estamos en terreno conocido, nos limitamos a hacer en síntesis un servicio para ejercitar la memoria:

-Recordamos que no hay formación sin receptividad, que es la que caracteriza al discípulo. La receptividad da al alumno la categoría de discípulo. Consecuentemente, no hay formación sin discípulo, es decir, sin receptividad. El discipulado está en el corazón de la formación.

-Recordamos que en la formación sacerdotal no hay formación inicial ni permanente sin discípulo. Y si PDV 71 de forma explícita habla de continuidad, de la intrínseca relación entre la «formación inicial» y la «formación permanente», y de la formación como un hecho unitario, el discipulado no puede desaparecer entre un momento y otro. El discipulado, aunque con rasgos y caracteres propios de cada momento, debe permanecer. Un Seminario que hoy no capacita al nuevo sacerdote para un discipulado posterior ha fracasado; $y$ se puede decir más, que ya está dificultando en gran medida el mismo futuro de la Formación integral Permanente de los sacerdotes. Creemos, por tanto, que uno de los objetivos fundamentales del Seminario actual ha de ser formar para un discipulado permanente. Si no llegamos a esto, podemos afirmar rotundamente que hemos fracasado

-Recordamos, también, la dificultad que hoy tiene el aceptar y vivir la actitud de discípulo, que llega a ser una manera de ser, y que sólo puede adoptarse desde unas referencias hondamente vividas. Las resistencias existen y son fuertes, pero pueden afrontarse.

20. Comisión Episcopal del Clero, La Formación permanente..., 348. 


\section{b) El discipulado y el sacerdote}

Adentrarnos en la espesura del discipulado de Jesús, que en parte es conocida para todos nosotros, es una experiencia siempre nueva, bella como la que más, y gozosa como ninguna. El discipulado del sacerdote es más que la receptividad y la acogida de la formación, es mucho más, es otra cosa; entra en su ser; es constitutiva del sacerdocio. El discipulado es constitutivo del sacerdocio. Ser sacerdote no es hacerse discípulo; es ser discípulo.

Este ser discípulo del sacerdote se entiende -y más bien, se vive- en razón de que se es sacerdote en Cristo sacerdote, es decir, que no nos hacemos sacerdotes por nuestra relación con Él, sino que somos sus sacerdotes en la relación suya con nosotros. Somos sacramento de Cristo Cabeza y Pastor y nos corresponde «actuar en su nombre». Ser sacramento - «actuar en su nombre»es un discipulado total, es ser su discípulo.

Con lo que acabamos de decir se entiende, en primer lugar, que no hay comprensión del sacerdocio al margen del discipulado más radical que cabe, $y$, en segundo lugar, que no hay vivencia del sacerdocio sin que el discipulado brote de lo profundo de la persona.

\section{c) El sacerdote discípulo y la Formación integral Permanente}

Si el discípulo es un modo de ser y el modo de ser del sacerdote está configurado por la misma identidad sacerdotal que incluye el discipulado, el discípulo entra dentro de la estructura de la persona del sacerdote, que siempre, inevitablemente, será discípulo. Esta configuración de la persona con discipulado incluye una antropología propia. El sacerdote es discípulo aunque deba ejercer de maestro. Se cumple en él lo de: «El Señor me ha dado lengua de discípulo... cada mañana me espabila el oído para que escuche como los discípulos» (Is 50, 4).

La incidencia en la Formación Permanente es del todo clara: No pueden plantearse por separado el discipulado en la Formación Permanente y el discipulado propio de ser sacerdote. Se trata de una misma realidad. El sacerdote, que es discípulo, lo es en la Formación permanente. Y se nota, porque el discipulado es de su antropología.

La consecuencia es notable: no es posible pensar en un planteamiento nuevo de Formación Permanente sin contar con el discipulado en el sacerdote, cuya presencia debe serle connatural. El espíritu de discipulado en el sacerdote condiciona la Formación Permanente tanto en su planteamiento como en su ejecución.

\section{Referencias imprescindibles en la Formación Permanente}

No podemos terminar este trabajo sin señalar los aspectos que necesariamente deben ser atendidos por la Formación Permanente del sacerdote. La Formación Permanente, tanto la que se imparte como la que se personaliza y vive, no puede hacerse, a nuestro parecer, al margen de estas coordenadas o referencias. 


\section{a) La identidad del sacerdote}

La referencia al ser del sacerdote no puede faltar ni en la formación inicial ni en la posterior o permanente; la identidad está presente, de una forma o de otra, en la formación. Esta presencia de la identidad en la formación puede verse desde dos perspectivas distintas. En la primera, se ve a la identidad sacerdotal como objeto de la Formación Permanente, es decir: es la Formación Permanente la que atiende a la identidad del sacerdote, es su objeto de atención; y lo hace clarificando, cuestionando, enriqueciendo y potenciando la visión que el sacerdote puede tener de su identidad, que de todo se necesita. En la segunda, la identidad está presente como principio activo de la Formación Permanente; es la fuente, el principio de donde surge la fuerza, la exigencia y el criterio de la Formación Permanente. Esta perspectiva está muy en línea con la tesis que cruza toda la Exhortación de PDV: «La identidad presbiteral, como principio estructurador e integrador de la persona del sacerdote».

Diríamos, como resumen, que la Formación Permanente debe partir de la identidad del sujeto y debe terminar en la identidad del sujeto; es decir, la identidad es el punto de partida de la Formación Permanente y es el término de llegada. Por lo que acabamos de decir, la referencia a la identidad del sacerdote es prioritaria en la Formación Permanente. Tal referencia necesita hoy ser subrayada.

\section{b) El Ministerio del sacerdote}

Sabemos muy bien que el hablar de la identidad del sacerdote nos está pidiendo que inmediatamente y sin pasar página hablemos del ministerio, porque no hay identidad del sacerdote sin ministerio. Consecuentemente, no puede faltar de la Formación Permanente la referencia al ministerio. Sabemos que la razón de la ordenación es el ministerio y se nos ha recordado en PDV 73 que el fin de la Formación Permanente es «ayudar al sacerdote a ser y a desempeñar su función en el espíritu y según el estilo de Jesús buen Pastor».

Reconocemos que es muy común ver que la Formación Permanente busque potenciar en el sacerdote su capacidad de acción pastoral en el momento actual, que tanto se necesita -como lo está indicando la preparación al Sínodo de Obispos en este año 2012 sobre La Nueva Evangelización-, pero no está de más recordar que es propio de la Formación Permanente ahondar en el sentido del Ministerio recibido, para que el sacerdote se vea en él y se goce en él.

\section{c) La referencia de la Iglesia}

La referencia a la Iglesia es también imprescindible en la Formación Permanente de los sacerdotes. La razón está en la relación que el sacerdote tiene con la Iglesia particular, y en ella con la Iglesia universal. Tal relación es estrechísima; tanto es así que es sacerdote en la Iglesia. Y así se explica que la crisis de Iglesia sea crisis de sacerdocio y que el cuestionamiento de la Iglesia sea cuestiona- 
miento del sacerdocio en ella, y así lo vive muchas veces el sacerdote. Pero hay algo más, el sacerdote cuenta con una eclesialidad propia, ya que le corresponde un triplex munus en la Iglesia, y en su ministerio ejerce la representatio Ecclesiae; lo cual implica una relación propia y peculiar con la Iglesia particular. La referencia a la Iglesia particular del sacerdote diocesano es hoy irrenunciable.

El papel de la Formación Permanente es clave en este tema. Podemos verlo poniéndola ante esta situación muy concreta. Lo obvio es que el sacerdote viva una eclesiología en la que quepa sin sobresaltos -es decir: sin forzar la eclesiología y sin forzar el sacerdocio- el ministerio sacerdotal que le da la misma Iglesia. ¿No resultaría extraño que el sacerdocio ministerial que el presbítero recibe de la Iglesia en la ordenación no encajara a continuación con la eclesiología que se vive y se presenta en el entorno? El papel de la Formación Permanente también en este punto es clave.

\section{d) La estructuración de la persona del sacerdote}

Señalamos, por fin, otro punto del que no puede prescindir la Formación Permanente de los sacerdotes. Una Formación integral Permanente que no atiende a la persona del sacerdote en su estructuración a lo largo del proceso de la vida es un sin sentido, una contradicción total. Después de esta afirmación, ¿se puede añadir algo más? Es suficiente con que recalquemos una vez más la integralidad de la Formación Permanente en todo el proceso de la vida y en la persona del sacerdote, teniendo muy presente, también, al mayor y al desvalido.

\section{Hacia una conclusión}

Estamos muy agradecidos a lo que la Formación Permanente nos ha dejado en sus largos años de andadura; lo hemos reconocido en las páginas anteriores. Gracias a su aportación hemos llegado a tener conciencia de lo que es la Formación Permanente y lo que no es; y nos ha quedado muy claro que ya no podemos pensar en el sacerdocio sin su Formación integral Permanente. Pero, al mismo tiempo, también ha quedado muy claro que hay que repensar la Formación Permanente de los curas. La tarea que nos espera no es la de una mera adaptación; es otra cosa. La visión del edificio de la Formación Permanente admite diversas perspectivas y distintas caras: que tiene apariencias de un buen ver, que está ya gastado y sin solidez, que su estado es un tanto ruinoso y pide derribo, y que hasta se encuentra en ruinas. Con este panorama, diverso según los ángulos de visión, no es peregrino opinar, sino de sentido común, que deberá hacerse otro edificio aprovechando elementos del anterior.

Desde lo que venimos diciendo, ponemos en la conclusión estos subrayados:

-Es impensable hoy el sacerdocio sin la Formación integral Permanente.

- La identidad del sacerdote, en toda su amplitud y riqueza, es la primera referencia de la Formación integral Permanente. 
-La Formación integral Permanente incluye dos planos: la formación integral personalizada, y la formación integral más general, que es para todos. No deben confundirse ambos planos.

-La Formación integral Permanente personalizada es de todo sacerdote. A todo sacerdote, pertenezca a grupos determinados o no, le corresponde esta formación integral personalizada.

-La Formación integral Permanente no puede perder de vista, o mejor, debe tener como punto de partida el mismo Seminario, ya que la formación en él configura la Formación Permanente posterior dentro de la Iglesia Particular.

- La institucionalización de la Formación integral Permanente es necesaria. En este punto nos referimos principalmente a los sacerdotes diocesanos, sólo diocesanos; porque los que pertenecen a grupos definidos, tienen ya institucionalizada su formación.

-Cae por su propio peso que sin formador o acompañante no hay Formación integral Permanente. Puede ser que lo que se da por supuesto es de lo que más se carece. Sin maestro y sin discípulo no tenemos formación.

- ¿Y no queda al descubierto que el presbiterio necesita un nuevo planteamiento en la Iglesia particular? Es el gran reto ante el que hoy nos enfrentamos.

Miramos con gran esperanza esta etapa nueva del sacerdocio ministerial en nuestras Iglesias diocesanas. 